\title{
Urethral Extensibility Applied to Urethral Advancement
}

\author{
Bahir Sabah Allawi ${ }^{1 *}$, Alaa H. Al-Farhan², Rafid F. Al-Hussaini ${ }^{1}$ \\ ${ }^{1}$ Ministry of Higher Education and Scientific Research, College of Medicine, University of Babylon, Hillah, Iraq; ${ }^{2}$ Department of \\ Surgery, Basrah Medical College, Basrah, Iraq
}

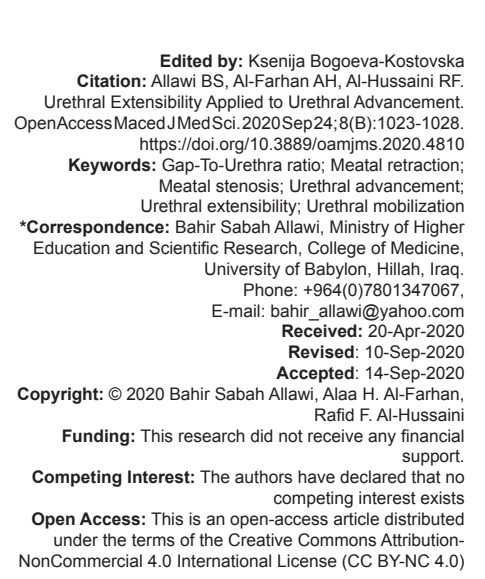

\begin{abstract}
BACKGROUND: The male urethra in humans has a large capacity to extend under traction. This property is the main principle of the urethral advancement technique.

AIM: We aimed to determine the safe limits of urethral mobilization and extensibility for reconstructive surgery of distal to midshaft hypospadias by applying urethral extensibility on the urethral advancement technique. Also, we wanted to evaluate the variable application of the gap-to-urethra $(G: U)$ ratio from a cadaver to a live human body.

METHODS: From November 2004 to February 2006, 20 boys aged from 2 to 16 years old underwent repair of midshaft to glanular hypospadias by urethral advancement technique. The ratio of $\mathrm{G}: \mathrm{U}$ proposed for a safe limit of urethral extensibility (measured from normal, fresh human cadaveric urethras) applied to know if its application can help in decreasing the rate of late complications. The mean follow-up period was 10 months, which ranged from 4 to 16 months.

RESULTS: Late complications were as follows: $25 \%$ meatal retraction (MR), $15 \%$ meatal stenosis (MS), and $5 \%$ fistula. Besides, MR and MS late complications associated with urethral mobilization of G:U ratio of less than $73 \%$.

CONCLUSION: The more the approximation in the application of the G:U ratio, the less the rate of MS and MR Besides, urethral mobilization to the base of the penis helps to decrease the frequency of MS and MR. Furthermore, the type of complication, MR or MS, in urethral advancement correlates with the extent of urethral mobilization.
\end{abstract}

\section{Introduction}

Hypospadias is a failure in the development of the urethral spongiosum, ventral prepuce, and normal embryological correction of penile curvature [1]. It is one of the common congenital anomalies; it occurs in 1 of 200-300 live births [1], [2]. Besides, distal hypospadias constitutes $75 \%$ of the cases [2].

The etiology of hypospadias is unknown. However, combinations of environmental, endocrine, and genetic factors, such as a defect in the epidermal growth factor in the ventral penis, are involved in its development [3].

The only treatment option for hypospadias is surgery. The goals of the surgical repair are appropriate reconstructions of the anatomically abnormal position of the meatus and urethra into the normal, as well as adequate functional outcome and acceptable esthetic appearance [1], [4], [5]. In the literature, more than 300 different surgical techniques had been described, but no consensus has been reached on a standard technique that gives satisfactory results [6]. Thus, the surgeon should choose the specific technique of repair for the particular anomaly of the hypospadias.
Despite the accepted cosmetic results of some procedures using hinged ventral penile skin flap, like Mathieu procedure, they have the problem of weak blood supply and, therefore, dehiscence may occasionally occur [7]. The original meatal advancement and glanuloplasty has primary drawbacks due to meatal retraction (MR) and meatal stenosis (MS) [6]. Besides, tubularized incised plate urethroplasty (TIP) had got more popularity for correction of distal hypospadias; however, its results depend on the characters of the urethral plate, together with an incidence of disruption, fistula, and MS [8]. Furthermore, the urethral advancement and glanuloplasty (UAGP), also known as sleeve advancement [9] and urethral mobilization [10], has fewer complications. They can be performed in one stage, commonly used to repair anterior hypospadias (distal shaft, coronal, and glanular) with or without chordee, and most reports confirm that no fistula has been observed [10].

The blood supplies of the urethra are antegrade from the posterolateral bulbar vessels and retrograde from the glans. However, in hypospadias due to poor development of the distal spongiosum, there is no efficient distal retrograde element of urethral vascularization [11] Therefore, adequate antegrade blood flow is considered as one of the crucial factors in the results of reconstructive 
surgeries [11]. Furthermore, the urethra and corpus spongiosum are supplied by the bulbourethral artery, which courses along with them, and this makes complete mobilization of the urethra with the corpus spongiosum possible without compromising their vascularity [12].

Tunica albuginea is a sturdy fibrous shield of the thick adventitia and its collagen fibers that envelop and provide the rigid covering of the erectile tissues of corpora cavernosa and spongiosum (Figure 1). Besides, it has two layers; inner circular and outer longitudinal layers; these are proximally inserted into the pubic bones [13]. Although it is limiting the penile extensibility, it is necessary to occlude the venous drainage of corpora cavernosa to support the penis during the erection. However, the tunica albuginea around the corpus spongiosum is composed of only the circular layer [13]. Thus, it permits more extensibility to the urethra and the corpus spongiosum in comparison to the corpora cavernosa, and this is the principle of urethral advancement procedure [12].

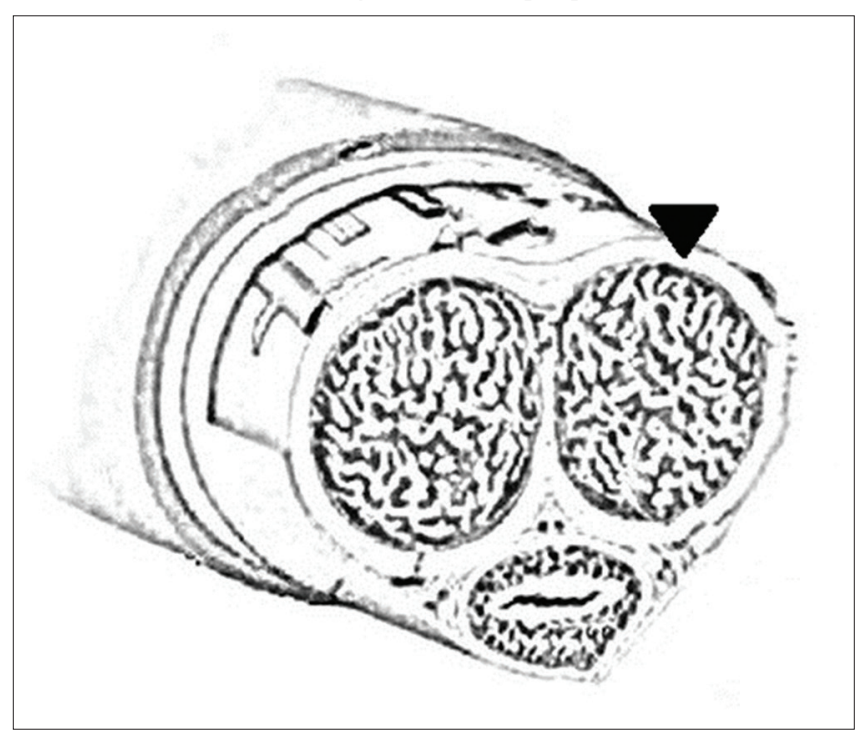

Figure 1: Structures of tunica albuginea (arrowhead)

Histologically, the anterior urethra is composed of an extracellular matrix that is arranged uniquely. The elastic fibers and smooth muscle cells are significant components of the extracellular matrix, in addition to its spongy feature; they form the male urethra with a large capacity to extend under traction [13].

Urethral extensibility is the percent variation in maximal constant traction length and urethral length at rest [13]. This extensibility is the basis of the urethral advancement technique to overcome urethral defects by complete freeing dissection, that is, mobilization of the urethra from the surrounding penile tissues and stretching it for distal advancement. While excess mobilization of the urethra is not desirable, the success rate of this surgical technique depends on the ability to form free tension anastomosis of the new meatus with the stretched urethra [13].

Urethral anastomosis under much tension can cause complications such as penile curvature or chordee, ischemia of the anastomosis site or even necrosis, MR, MS, and dehiscence. Besides, extensive urethral mobilization can cause injury to the spongiosclerosis, the urethral blood supply, or vascular erectile dysfunction [14]. Furthermore, overstretching of the male urethra decreases its lumen and may cause high bladder pressure during voiding according to the law of Laplace [15]. Therefore, the application of urethral extensibility and its safe anatomical limit in reconstructive surgery should avoid these complications and the need for more sophisticated techniques. This limit should be a general gap-to-normal urethra (G:U) ratio of $1: 4$; when $G$ is the distance from the meatus to the glans tip and $U$ is the length of the urethral mobilization [13]. However, age-related variations of urethral extensibility should be considered because the ratio in older patients is higher than in younger patients, as structural changes in the extracellular matrix of the penis with aging will decrease its extensibility [13]. Thence, the suggested limits should be adjusted to the patient's age (Table 1). These limits were the results of the study on the extensibility of fresh human cadaveric urethras [13].

Table 1: Changes in the length of normal urethra necessary to overcome the gap by patient's age

\begin{tabular}{ll}
\hline Age (years) & G:U ratio \\
\hline 1 & $1: 3.2$ \\
10 & $1: 3.4$ \\
20 & $1: 3.7$ \\
30 & $1: 4.0$ \\
40 & $1: 4.5$ \\
50 & $1: 5.0$ \\
60 & $1: 5.7$ \\
70 & $1: 6.6$ \\
\hline G:U: Gap to urethral. &
\end{tabular}

Extrapolating these results in cadavers to live urethras may have some limitations. However, they studied macroscopically normal, fresh cadaveric urethras, which are regarded as the ideal available samples to answer the clinical question of how far the male urethra can be stretched before causing penile curvature [13].

\section{Patients and Methods}

From November 2004 to February 2006, a total of 20 patients with midshaft to glanular hypospadias with or without chordee had been operated by urethral advancement procedure. The patients were selected from Babylon city, Iraq. Further, the age of the patients ranged from 2 to 16 years old, with a mean of 5.9 years. Most of the patients were aged $2-5$ years $(60 \%)$ followed by ages of $6-10$ years $(30 \%)$ and $>10$ years (10\%).

The degree of urethral advancement in each patient was recorded by measuring the gap between the meatus and the glans tip $(G)$ and the length of urethral mobilization $(U)$, then calculated as in percentage to the 
$\mathrm{G}: U$ ratio according to the age of the patient. In some of the patients, urethra mobilized less, the same or more than the ratio written in Table 1 , which were to evaluate the variable application of the $G: U$ ratio from the cadaver to the live human body.

\section{Surgical procedure}

Once the patient anesthetized under general anesthesia in the supine position and after sterilization and toweling the operative field, a traction 3-0 silk suture with a round needle was placed high in the dorsum of the glans. The distance between the hypospadiac meatus and the tip of the glans penis was measured and recorded, and urethral dilators were used to improve MS (Figure 2).

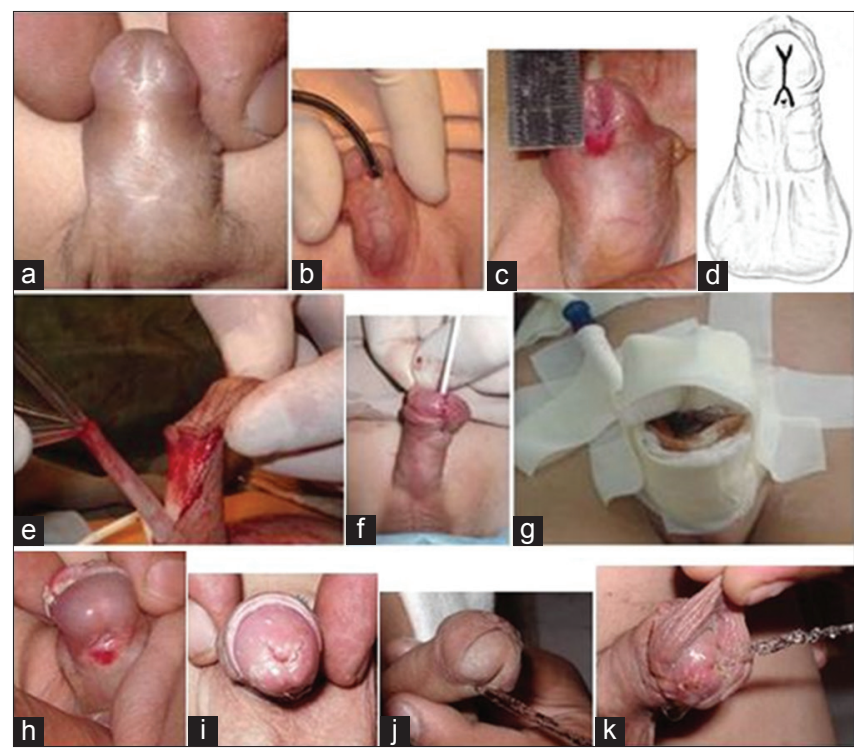

Figure 2: Urethral advancement glanuloplasty. (a) Subcoronal hypospadias with meatal stenosis (b), meatal dilatation, (c) measurement of the distance to the tip, (d) perimeatal diamond and $Y$-shaped glans marking and incisions, (e) dissection and mobilization of the urethra using a catheter with dorsal wide meatotomy up to corpus spongiosum and the distal urethra advancement and suturing to midline $V$ flap of glans tissue, (f) post-correction meatus at tip, (g) dressing, (h) pre-operative subcoronal meatus, (i) post-operative meatus at glans tip, (j) pre-operative ventral urine stream, (k) postoperative straight urine stream

Marking drew by methylene blue was done (Figure 2). The incisions were made after the application of a tourniquet (Figure 2), then an incision performed deeply in the ventral furrow of glans to the distal corpora cavernosa, and glans wings were spatulated laterally but not more dorsally because this may have compromised the vascularity of the glans wings.

Elevation of the skin from distal urethra performed with great care, which is almost translucent and thin walled. Then, ventral skin or the whole penile skin was dissected in a sleeve-like pattern to release cutaneous chordee distally, and superficial tethers along the skin of the shaft proximally, and then the skin of the shaft degloved down to the penoscrotal junction. Most mild degrees of chordee were released after this step, but any remaining chordee was corrected by excising any dysplastic tissues. After that, the urethral meatus was circumscribed using a scissor laterally, and the dissection was deepened down to the corpora cavernosa. Then, the avascular plane that is between the corpora cavernosa and the corpus spongiosum was created and continued proximally.

The urethra was mobilized using the indwelling catheter for gentle counter traction (Figure 2). The urethra assembled as needed to obtain a tension-free anastomosis between the glans of the taut phallus and the advanced urethra. Meatotomy was then done by widely spatulating the dorsal meatus. Furthermore, the glanular $\mathrm{V}$ shape was dissected as a flap of glanular mucosa and some supporting glandular tissue. The mobilized urethra with the surrounding corpus spongiosum advanced between the spatulated glans wings to the base of the $V$ flap, then the end of the meatotomy fixed to the tip of the $\mathrm{V}$ flap. Absorbable sutures (5-0) were used for the fixation of the lateral points of the meatotomy to fix it to the bases of the $V$ flap. Finally, further anastomosed of the urethral meatus to the glans was performed with interrupted sutures.

The two glans wings were closed over the urethra in two layers; the first layer incorporating the deep glanular tissue and corpus spongiosum together, then the glans wings were approximated to provide a generous mucosal cuff around the urethra. Besides, care was taken to ensure that the glans closure was not tight around the urethra. After that, the meatal anastomosis was completed by placing ventral lateral sutures which were completed the meatoglanuloplasty (Figure 2). Finally, the tourniquet was released; hemostasis secured by bipolar electrocautery, and closure of the shaft skin wound was performed by 4-0 absorbable sutures.

Topical antibiotics and non-adherent first layer sandwiched by the second layer of dry gauze dressing were then applied with care to avoid excessive compression (Figure 2).

The patients discharged home the next day. The urinary indwelling catheter removed after 10-14 days, and the patients continued on urethral dilatation by urethral stents.

The patients were then kept under follow-up every month with particular emphasis on reporting any change in the urine stream direction, meatal site, meatal size, glans shape, recurrent chordee, and erection. Further, the mean follow-up period was 10 months, which ranged from 4 to 16 months.

\section{Results}

In 5 patients $(25 \%)$, the hypospadias had associated with a mild degree of ventral chordee. 
The most common late complication was MR (Table 2).

Table 2: Frequencies of late complications

\begin{tabular}{lll}
\hline Late complication & Frequency & Percent $^{*}$ \\
\hline MS & 3 & 15 \\
MR & 5 & 25 \\
Fistula & 1 & 5 \\
Abnormal stream & 2 & 10 \\
\hline MR: Meatal retraction, MS: Meatal stenosis. ${ }^{*}$ These percentages were calculated separately.
\end{tabular}

The late complications were associated with urethral mobilization of the $\mathrm{G}: \mathrm{U}$ ratio of less than $73 \%$ (Table 3).

Table 3: The proposed G:U ratio according to the age applied to the degree of mobilization and its associated incidence of MS and MR in each group

\begin{tabular}{|c|c|c|c|c|c|c|c|}
\hline Group & $\begin{array}{l}\text { Age } \\
\text { (years) }\end{array}$ & $\begin{array}{l}\mathrm{G}: \mathrm{U} \\
\text { ratio }\end{array}$ & $\begin{array}{l}\text { Meatus tip } \\
\text { gap }(\mathrm{cm})\end{array}$ & $\begin{array}{l}\text { Length of } \\
\text { mobilization }(\mathrm{cm})\end{array}$ & $\begin{array}{l}\% \text { of mobilization } \\
\text { according to G:U ratio }\end{array}$ & MS & MR \\
\hline \multirow[t]{6}{*}{$\mathrm{T}$} & 2 & $1: 3.2$ & 0.9 & 1.5 & 53.5 & $\sqrt{ }$ & - \\
\hline & 3 & $1: 3.2$ & 1.2 & 1.5 & 39.4 & $\sqrt{ }$ & $\sqrt{ }$ \\
\hline & 5.3 & $1: 3.2$ & 1.5 & 2 & 41.6 & - & - \\
\hline & 7 & $1: 3.2$ & 1.5 & 3.5 & 72.9 & - & $\sqrt{ }$ \\
\hline & 14 & $1: 3.4$ & 1.5 & 2.5 & 49 & - & - \\
\hline & 16 & $1: 3.4$ & 2 & 3 & 44.1 & - & $\sqrt{ }$ \\
\hline \multirow[t]{6}{*}{ II } & 4.4 & $1: 3.2$ & 1.2 & 1.5 & 39.4 & $\sqrt{ }$ & $\sqrt{ }$ \\
\hline & 4.5 & $1: 3.2$ & 1 & 2 & 62.5 & - & - \\
\hline & 5 & $1: 3.2$ & 0.4 & 1.5 & 115.3 & - & - \\
\hline & 5.6 & $1: 3.2$ & 1.3 & 2.5 & 59.5 & - & - \\
\hline & 7 & $1: 3.2$ & 1.3 & 2 & 47.6 & - & - \\
\hline & 9 & $1: 3.2$ & 1 & 1.2 & 37.5 & - & - \\
\hline \multirow[t]{8}{*}{ III } & 3 & $1: 3.2$ & 1 & 3 & 93.7 & - & - \\
\hline & 3.8 & $1: 3.2$ & 1.5 & 2.5 & 52 & - & - \\
\hline & 4 & $1: 3.2$ & 1.2 & 3.3 & 86.8 & - & - \\
\hline & 4 & $1: 3.2$ & 1 & 2.5 & 78.1 & - & - \\
\hline & 4.6 & $1: 3.2$ & 1.4 & 2.1 & 46.6 & - & $\sqrt{ }$ \\
\hline & 6 & $1: 3.2$ & 1.3 & 4.5 & 107.1 & - & - \\
\hline & 7 & $1: 3.2$ & 1 & 4 & 125 & - & - \\
\hline & 7 & $1: 3.2$ & 1.3 & 4 & 95.2 & - & - \\
\hline
\end{tabular}

\section{Discussion}

In the current study, the mean age at surgery was 5.9 years; it reflects the late presentation of the patients for asking to solve the problem. Therefore, we need to inform our society about this common congenital anomaly and let them know they need for earlier consultation to have enough time for finishing the management before school age.

The overall late complications rate in the current study was $30 \%$, which is less than the complication rate after urethral lengthening procedures (77\%) and the overall complication rate $(54 \%)$, but higher than the complication rate after urethral advancement (22\%) reported by Nuininga et al. [16]. The study of Nuininga et al. [16] handled different types of single-stage hypospadias repair procedures and followed them up for at least 10 years. Moreover, long-term follow-up after hypospadias repair is essential [16]; therefore, the overall complication rate in the current study may have been more if we followed the patients for a more extended period.

The complications were varied according to the type of procedures performed. For example, the study of Arajy [17] on 68 patients to evaluate UGPI procedure in distal hypospadias with no or minimal chordee for 8 years follow-up found $9 \% \mathrm{MR}, 14.7 \% \mathrm{MS}$, and $5.8 \%$ fistula. Furthermore, MR constituted the majority of complications in the current study (Table 2), and we think that it was related to the degree of urethral mobilization for advancement. In contrast to the lower rates of fistula complication in the studies mentioned above, the reported incidence of fistula formation in published articles was higher depending on the severity of the initial deformity; it varied between 7 and $23 \%$ for different procedures [16]. Thus, the UAGP provides the most watertight urethroplasty in hypospadias repair.

For researching purposes, the patients were divided into three groups; Groups I, II, and III, with different range in urethral mobilization, although all of them had the same principle of urethral mobilization and advancement to the glans tip (Table 3). The ratio of $G: U$ proposed for the safe limit of urethral extensibility [13] was introduced in this study to find out if its application can help in decreasing the incidence of MR and MS (Table 3).

The range of urethral mobilization according to the G:U ratio in Group I was $39.4-72.9 \%$, which is the least of the other groups, and the rate of MR was $50 \%$, and MS was $33.3 \%$. While this range in Group III was $46.6-125 \%$, which was the highest as compared to the other groups and the least rate of MR $12.5 \%$ occurred, but no patient was afflicted with MS (Table 3). Hence, the more the extent of urethral mobilization, the fewer incidences of MR and MS occurred. It can be explained by imagining the penile urethra as a compressed spiral tube in a resting position with the ability of the extensibility. If it is freed from only the distal part and extended to advance to the tip, there will be more tension on the tip and leads to both MR and MS complications. While if it is completely freed and extended, there will be an excess length of the tube with less or no tension on the tip; thence, no complications will develop.

On the other hand, extensive urethral dissection may lead to injury of spongiosclerosis, the urethral blood supply, or vascular erectile dysfunction. Besides, overstretching of the male urethra decreases its lumen and may cause high bladder pressure during voiding according to the law of Laplace [15]. Although the previously mentioned complications were not reported in the current study, long-term follow-up is needed to detect them. Therefore, the restriction in the application of the $\mathrm{G}: U$ ratio may help to prevent these complications; from under application of the ratio, that is, less dissection of the urethra, or over application, that is, extensive dissection. Thus, the implementation of the $G: U$ ratio may help to determine the safe anatomical limit of urethral mobilization; on the other hand, urethral mobilization to the base of the penis will help to decrease the incidence of MS and MR.

The types of complications, MR or MS, in urethral advancement correlate with the extent of 
urethral mobilization. When there was more than $73 \%$ mobilization of the G:U ratio, no MR or MS reported (Table 3); while at $72.9 \%$ mobilization, MR occurred in a patient with midshaft hypospadias and underwent urethral advancement, and after dissection of his urethra, it was found that his urethra was short although it could reach to the tip with tension on the neomeatus site. While at $53 \%$ mobilization and below, the patient develops MS only because inadequate urethral mobilization can only cause tension on the meatus, which in the long term causes the meatus contraction into a smaller size and, in turn, leads to MS (Figure 3). This feature can be found in the physical property of elastic tubes; it will decrease in its caliber when it is stretched.

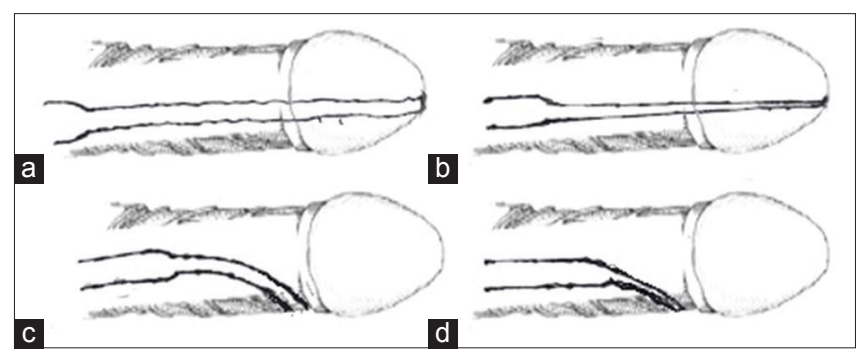

Figure 3: Diagram of the urethra (a) no complications with adequate and complete urethral mobilization, (b) meatal stenosis, (c) meatal retraction, (d) meatal stenosis and retraction together At $44 \%$ and $46 \%$, the mobilization is inadequate to the degree to cause tension, which is enough to lead to meatal and urethra regression, and when the tension was relieved on the meatus, no MS was reported. While at $39 \%$ mobilization, the tension on the anastomosis was severe enough to causes both MR and MS. This is because of the sustained tension on the meatus even after meatal regression to the more proximal site, and this leads to the narrowing of the meatus and development of MS

At $44 \%$ and $46 \%$, the mobilization is inadequate to the degree to cause tension, which is enough to lead to meatal and urethra regression, and when the tension was relieved on the meatus, no MS was reported. While at $39 \%$ mobilization, the tension on the anastomosis was severe enough to causes both MR and MS. This is because of the sustained tension on the meatus even after meatal regression to the more proximal site, and this leads to the narrowing of the meatus and development of MS.

\section{Conclusion}

The incidence of MR and MS is related to the degree of urethral mobilization for advancement; above $73 \%$ mobilization of the G:U ratio, no MR or MS reported and the more the approximation in the application of the $\mathrm{G}: U$ ratio, the less incidence of $M S$ and MR occurred. Restriction in implementing the $\mathrm{G}: \mathrm{U}$ ratio will help prevent the complications of both under or over application (less dissection or extensive dissection, respectively) of the ratio. On the other hand, urethral mobilization to the base of the penis helps to decrease only the incidence of MS and MR, but it may cause other late complications that not reported in this shortterm study. Furthermore, the types of complications, MR or MS, in urethral advancement correlate with the extent of urethral mobilization.

Hence, the application of the G:U ratio may help to determine the safe anatomical limit of mobilization of the urethra.

The main limitations of our study are the shortterm follow-up period and small sample size. Therefore, we suggest performing new studies on the same topic on larger samples and follow the patients for more extended periods.

\section{Acknowledgment}

We are thankful for Tropk for Computer and Scientific Research for their help in the edition and proofreading of this manuscript.

\section{References}

1. Baskin LS, Ebbers MB. Hypospadias: Anatomy, etiology, and technique. J Pediatr Surg. 2006;41(3):463-72. PMid:16516617

2. Alkan M, Oguzkurt P, Ezer SS, Ince E, Hicsonmez A. Evaluation of the results of eccentric circummeatal-based flap with combined limited urethral mobilization technique for distal hypospadias repair. J Pediatr Urol. 2008;4(3):206-9. https://doi. org/10.1016/j.jpurol.2007.11.008

3. Mouriquand PD, Mure PY. Hypospadias. In: John G, Richard R, Pierre $M$, editors. Pediatric Urology. $2^{\text {nd }}$ ed. Philadelphia, PA: Saunders; 2009. p. 713-27.

4. Ratan SK, Ratan J, Rattan KN. Is tubularization of the mobilized urethral plate a better alternative to tubularization of an incised urethral plate for hypospadias repair? Pediatr Surg Int. 2009;25(2):185-90. https://doi.org/10.1007/s00383-008-2312-8 PMid:19096854

5. Sensöz O, Ortak T, Baran CN, Unlü RE. Anew technique for dista hypospadias repair: Advancement of a distally deepithelialized urethrocutaneous flap. Plast Reconstr Surg. 2003;112(3):840-3. https://doi.org/10.1097/01.prs.0000070179.92405.c9 PMid:12960867

6. Elsayed ER, Khalil S, Abd Samad K, Abdalla MM. Evaluation of distally folded onlay flap in repair of distal penile hypospadias. J Pediatr Urol. 2012;8(1):103-7. https://doi.org/10.1016/j. jpurol.2010.09.008 PMid:20889383

7. Mathieu P. Traitement en un temps de l'hypospade balanique et juxta-balanique. J Chir. 1932;39:481-7.

8. Hammouda HM, El-Ghoneimi A, Bagli D, McLorie G, Khoury AE. Tubularized incised plate repair: Functional outcome after intermediate followup. J Urol. 2003;169(1):331-3. https://doi. 
org/10.1016/s0022-5347(05)64120-1

PMid:12478185

9. Murphy JP. Hypospadias. In: Ashcraft KW, Murphy JP, Sharp RJ, Sigalet DL, Snyder CL, editors. Pediatric Surgery. $3^{\text {rd }}$ ed. Philadelphia, PA: Saunders; 2000. p. 763-79.

10. Belman AB. Hypospadias and chordee. In: Belman AB, King LR, Kramer SA, editors. Clinical Pediatric Urology. $4^{\text {th }}$ ed. London: Martin Dunitz; 2002. p. 1061-88.

11. Roodsari SS, Mulaeian M, Hiradfar M. Urethral advancement and glanuloplasty with $\mathrm{V}$ flap of the glans in the repair of anterior hypospadias. Asian J Surg. 2006;29(3):180-4. https://doi. org/10.1016/s1015-9584(09)60083-x PMid:16877221

12. Chang JA, Lue TF. Surgical anatomy of the penis and erectile bodies1. Atlas Urol Clin North Am. 2002;10(2):119-25.

13. Da Silva EA, Sampaio FJ. Urethral extensibility applied to reconstructive surgery. J Urol. 2002;167(5):2042-5.

PMid:11956434
14. Coursey JW, Morey AF, McAninch JA, Summerton DJ, Secrest C, White $\mathrm{P}$, et al. Erectile function after anterior urethroplasty. J Urol. 2001;166(6):2273-6. https://doi. org/10.1016/s0022-5347(05)65549-8

PMid:11696750

15. Plevnik S, Janez J. Pressure-distention relation in male obstructed urethra. Urology. 1981;17(4):393-7. https://doi. org/10.1016/0090-4295(81)90281-8

PMid:7222341

16. Nuininga JE, DE Gier RP, Verschuren R, Feitz WF. Long-term outcome of different types of 1-stage hypospadias repair. J Urol. 2005;174(4 Pt 2):1544-8. https://doi.org/10.1097/01. ju.0000179255.55848.50

PMid:16148649

17. Arajy ZY. V-glanuloplasty and urethral advancement for distal hypospadias. Iraqi Postgrad Med J. 2004;3:131-6. 